\title{
Algebraic independence of the values of power series with unbounded coefficients
}

\author{
Kaneko Hajime
}

\begin{abstract}
Many mathematicians have studied the algebraic independence over $\mathbb{Q}$ of the values of gap series, and the values of lacunary series satisfying functional equations of Mahler type. In this paper, we give a new criterion for the algebraic independence over $\mathbb{Q}$ of the values $\sum_{n=0}^{\infty} t(n) \beta^{-n}$ for distinct sequences $(t(n))_{n=0}^{\infty}$ of nonnegative integers, where $\beta$ is a fixed Pisot or Salem number. Our criterion is applicable to certain power series which are not lacunary. Moreover, our criterion does not use functional equations. Consequently, we deduce the algebraic independence of certain values $\sum_{n=0}^{\infty} t_{1}(n) \beta^{-n}, \ldots, \sum_{n=0}^{\infty} t_{r}(n) \beta^{-n}$ satisfying

$$
\lim _{n \rightarrow \infty, t_{i-1}(n) \neq 0} \frac{t_{i}(n)}{t_{i-1}(n)^{M}}=\infty \quad(i=2, \ldots, r)
$$
\end{abstract}

for any positive real number $M$.

\section{The transcendence of the values of power series with bounded coefficients}

We introduce notation which we use throughout this paper. Let $\mathbb{N}$ (resp. $\mathbb{Z}^{+}$) be the set of nonnegative integers (resp. positive integers). For a real number $x$, we denote the integral and fractional parts of $x$ by $\lfloor x\rfloor$ and $\{x\}$, respectively. We use the Landau symbols $o, O$, and the Vinogradov symbols $\gg, \ll$ with their regular meanings. For a sequence of integers $\boldsymbol{t}=\left(t_{n}\right)_{n=0}^{\infty}$, put

$$
\mathcal{S}(\boldsymbol{t}):=\left\{n \in \mathbb{N} \mid t_{n} \neq 0\right\}
$$

and

$$
f(\boldsymbol{t} ; X):=\sum_{n=0}^{\infty} t_{n} X^{n}
$$

Key words and phrases: algebraic independence, Pisot numbers, Salem numbers. 2010 Mathematics Subject Classification: primary 11J99; secondary 11K16, 11K60. 
Note that if $t_{n} \geq 0$ for any $n$ and if $t_{n} \in\{0,1\}$ for any sufficiently large $n$, then (1.1) is rewritten as

$$
f(\boldsymbol{t} ; X)=\sum_{m=0}^{\infty} X^{v_{m}},
$$

where $\left(v_{m}\right)_{m=0}^{\infty}$ is a sequence of nonnegative integers satisfying $v_{m+1}>v_{m}$ for any sufficiently large $m$. Let $\mathcal{A}$ be a nonempty subset of $\mathbb{N}$. Set

$$
\lambda(\mathcal{A} ; R):=\operatorname{Card}\{[0, R) \cap \mathcal{A}\}
$$

for any $R \geq 1$, where Card denotes the cardinality.

In this paper, we study arithmetical properties of the values $x=f(\boldsymbol{t} ; \alpha)$ for a fixed algebraic number $\alpha$ with $0<|\alpha|<1$. In this section, we review known results of the transcendence of such values in the case where the coefficients are bounded.

In particular, if $\alpha$ is a real number with $0<\alpha<1$, put $\beta:=\alpha^{-1}$. In this paper, an infinite series of the form

$$
x=f\left(\boldsymbol{t} ; \beta^{-1}\right)=\sum_{n=0}^{\infty} t_{n} \beta^{-n},
$$

where $\boldsymbol{t}=\left(t_{n}\right)_{n=0}^{\infty}$ is a sequence of integers, is called a $\beta$-representation of $x$. The $\beta$-expansion of $x$, introduced by Rényi [17], is a $\beta$-representation computed by the greedy algorithm. Let $T_{\beta}:[0,1) \rightarrow[0,1)$ be the $\beta$-transformation defined by $T_{\beta}(x):=$ $\{\beta x\}$ for $x \in[0,1)$. Then the $\beta$-expansion of $x \in[0,1)$ is denoted by

$$
x=\sum_{n=1}^{\infty} t_{n}(\beta ; x) \beta^{-n},
$$

where $t_{n}(\beta ; x)=\left\lfloor\beta T_{\beta}^{n-1}(x)\right\rfloor$ for $n \geq 1$.

In the rest of this section, $\boldsymbol{v}=\left(v_{m}\right)_{m=0}^{\infty}$ denotes an ultimately increasing sequence of nonnegative integers. Recall that $\beta>1$ is a Pisot number if $\beta$ is an algebraic integer whose conjugates except itself have absolute value less than 1 . In particular, any integer greater than 1 is a Pisot number. Moreover, an algebraic integer $\beta>1$ is a Salem number if the conjugates of $\beta$ except itself have absolute values not greater than 1 and if $\beta$ has at least one conjugate with absolute value 1 .

Adamczewski [1] showed that if

$$
\limsup _{m \rightarrow \infty} \frac{v_{m+1}}{v_{m}}>1
$$


then $\sum_{m=0}^{\infty} \beta^{-v_{m}}$ is transcendental for any Pisot or Salem number $\beta$. In the case where $\alpha$ is a general algebraic number with $0<|\alpha|<1$, Corvaja and Zannier [7] showed that if

$$
\liminf _{m \rightarrow \infty} \frac{v_{m+1}}{v_{m}}>1
$$

then $\sum_{m=0}^{\infty} \alpha^{v_{m}}$ is transcendental.

However, it is difficult to study the transcendence in the case where

$$
\lim _{m \rightarrow \infty} \frac{v_{m+1}}{v_{m}}=1 \text {. }
$$

We review known results on the transcendence of certain values $\sum_{m=0}^{\infty} \beta^{-v_{m}}$ satisfying (1.4) in the case where $\beta$ is a Pisot or Salem numbers. The results are obtained by using the partial results on the normality of the $\beta$-expansions of algebraic irrational numbers. For instance, consider the case where $\beta=b$ is an integer greater than 1. Then Borel [4] conjectured that all algebraic irrational numbers are normal in base- $b$, which is still open. If Borel's conjecture is true, then we have the following: if

$$
\limsup _{m \rightarrow \infty} \frac{v_{m}}{m}=\infty
$$

then $\sum_{m=0}^{\infty} b^{-v_{m}}$ is transcendental.

Let $x \in[0,1)$ be an algebraic irrational number. For a positive integer $N$, let $\mu(b, x ; N)$ be the number of nonzero digits in the first $N$ digits of $x$ in base- $b$. In the case of $b=2$, Bailey, Borwein, Crandall, and Pomerance [3] gave lower bounds for $\mu(2, x ; N)$ with $N \geq N_{0}$, where $N_{0}$ is an ineffective positive constant. Modifying the proof of the above results, Adamczewski, Faverjon [2], and Bugeaud [5] independently gave effective versions of the lower bounds for $\mu(b, x ; N)$ with general integer $b \geq 2$. Lower bounds for the numbers of nonzero digits in $\beta$-representations of real numbers were also studied [10] and [11] in the case where $\beta$ is a Pisot or Salem number.

Using the lower bounds, we deduce for each Pisot or Salem number $\beta$ that if $\boldsymbol{v}=\left(v_{m}\right)_{m=0}^{\infty}$ satisfies

$$
\limsup _{m \rightarrow \infty} \frac{v_{m}}{m^{A}}=\infty
$$

for any positive real number $A$, then $\sum_{m=0}^{\infty} \beta^{-v_{m}}$ is transcendental (see Corollary 2.4 in [10]). If $\beta=b>1$ is an integer, then the transcendence of $\sum_{m=0}^{\infty} b^{-v_{m}}$ was essentially proved by Bailey, Borwein, Crandall, and Pomerance [3]. Consequently, we obtain the transcendence of $\sum_{m=0}^{\infty} \beta^{-v_{m}}$ for certain classes of $\sum_{m=0}^{\infty} \beta^{-v_{m}}$ satisfying (1.4). 
For instance, put, for any positive real $\nu$,

$$
\sigma_{1, \nu}(m):=\exp \left((\log m)^{1+\nu}\right)=m^{(\log m)^{\nu}} \quad(m=1,2, \ldots),
$$

and let

$$
\sigma_{2}(m):=\exp (\log m \log \log m)=m^{\log \log m} \quad(m=3,4, \ldots) .
$$

For any Pisot or Salem number $\beta$, we obtain the transcendence of

$$
\sum_{m=1}^{\infty} \beta^{-\left\lfloor\sigma_{1, \nu}(m)\right\rfloor} \text { and } \sum_{m=3}^{\infty} \beta^{-\left\lfloor\sigma_{2}(m)\right\rfloor}
$$

because $\left(\left\lfloor\sigma_{1, \nu}(m)\right\rfloor\right)_{m=1}^{\infty}$ and $\left(\left\lfloor\sigma_{2}(m)\right\rfloor\right)_{m=3}^{\infty}$ fulfill (1.5).

In what follows, we study the algebraic independence over $\mathbb{Q}$ of values $f(\boldsymbol{t} ; \alpha)$ for a fixed algebraic number $\alpha$ with $0<|\alpha|<1$ and distinct sequences $\boldsymbol{t}$. We also investigate the case where $\boldsymbol{t}$ is unbounded. In Section 2, we introduce known results on the algebraic independence of the values of power series satisfying certain lacunary assumption. In Section 3, we give main criterion for the algebraic independence in the case of $\alpha=\beta^{-1}$, where $\beta$ is a Pisot or Salem number. Using our criterion, we deduce the algebraic independence of real numbers including the values (1.6), which gives new examples of algebraic independence. We prove our criterion in Section 4.

\section{Algebraic independence of the values of lacunary series}

Let $\boldsymbol{t}=\left(t_{n}\right)_{n=0}^{\infty}$ be a sequence of integers such that $t_{n} \neq 0$ for infinitely many $n$ 's. Put

$$
\left\{n \in \mathbb{N} \mid t_{n} \neq 0\right\}=:\left\{w_{\boldsymbol{t}}(0)<w_{\boldsymbol{t}}(1)<\ldots\right\} .
$$

We say that $f(\boldsymbol{t} ; X)$ is a gap series if

$$
\lim _{m \rightarrow \infty} \frac{w_{\boldsymbol{t}}(m+1)}{w_{\boldsymbol{t}}(m)}=\infty .
$$

For instance, $\sum_{m=0}^{\infty} X^{m !}$ is a gap series. Moreover, we say that $f(\boldsymbol{t} ; X)$ is lacunary if

$$
\liminf _{m \rightarrow \infty} \frac{w_{\boldsymbol{t}}(m+1)}{w_{\boldsymbol{t}}(m)}>1 .
$$

For instance,

$$
\varphi_{k}(X):=\sum_{m=0}^{\infty} X^{k^{m}}
$$


is lacunary for any integer $k \geq 2$. In this section we introduce known results on the algebraic independence of the values of gap series, and lacunary series satisfying functional equations of Mahler type, respectively. In the rest of this section, $\alpha$ denotes an algebraic number with $0<|\alpha|<1$.

We consider the algebraic independence of the values of gap series. We first study the case where $f(\boldsymbol{t} ; X)$ has the form (1.2). Shiokawa [19] gave a criterion for the algebraic independence of gap series at algebraic points. Using his criterion, we obtain for each $\alpha$ that the set

$$
\left\{\sum_{m=0}^{\infty} \alpha^{(k m) !} \mid k=1,2, \ldots\right\}
$$

is algebraically independent, which generalizes the result by Schmidt [18].

Next we consider the case where $\boldsymbol{t}=\left(t_{n}\right)_{n=0}^{\infty}$ is unbounded. In particular, we study the algebraic independence of the set

$$
\left\{f^{(l)}(\boldsymbol{t} ; \alpha) \mid l=0,1, \ldots\right\},
$$

where $f^{(l)}(\boldsymbol{t} ; X)$ denotes the $l$-th derivative of $f(\boldsymbol{t} ; X)$. For any $m \in \mathbb{N}$, let

$$
A_{m}:=\max \left\{1,\left|t_{n}\right| \mid 0 \leq n \leq w_{\boldsymbol{t}}(m)\right\}
$$

and let $R$ be the convergence radius with $0<R \leq 1$. Then Cijsouw and Tijdeman [6] showed for any $\alpha$ with $|\alpha|<R$ that if

$$
\lim _{m \rightarrow \infty} \frac{w_{\boldsymbol{t}}(m+1)}{w_{\boldsymbol{t}}(m)+\log A_{m}}=\infty,
$$

then $f(\boldsymbol{t} ; \alpha)$ is transcendental. Nishioka [15] showed for each $\alpha$ with $|\alpha|<R$ that if (2.1) holds, then the set

$$
\left\{f^{(l)}(\boldsymbol{t} ; \alpha) \mid l=0,1, \ldots\right\}
$$

is algebraically independent.

Next, we investigate the algebraic independence of the values of lacunary series satisfying functional equations of Mahler type. For instance, if $k$ is an integer greater than 1 , then the series $\varphi_{k}(X)$ satisfy the following:

$$
\varphi_{k}\left(X^{k}\right)=\sum_{m=1}^{\infty} X^{k^{m}}=\sum_{m=0}^{\infty} X^{k^{m}}-X=\varphi_{k}(X)-X .
$$

Using the above relation, Mahler [12] showed the transcendence of $\varphi_{k}(\alpha)$ for any $\alpha$. In the rest of this section we introduce results on algebraic independence proved by Mahler's method. 
Nishioka [13] proved for any $\alpha$ that the set

$$
\left\{\varphi_{k}(\alpha) \mid k=2,3, \ldots\right\}
$$

is algebraically independent. More generally, Tanaka [20] showed for any positive real numbers $w_{1}, \ldots, w_{r}$ linearly independent over $\mathbb{Q}$ and any $\alpha$ that the set

$$
\left\{\sum_{m=0}^{\infty} \alpha^{\left\lfloor w_{i} k^{m}\right\rfloor} \mid i=1, \ldots, r, k=2,3, \ldots\right\}
$$

is algebraically independent.

We now consider the case where $\boldsymbol{t}=\left(t_{n}\right)_{n=0}^{\infty}$ is unbounded. We call a sequence $\left(w_{n}\right)_{n=0}^{\infty}$ of integers a linear recurrence if there exist $r \geq 1$ and $c_{1}, \ldots, c_{r} \in \mathbb{C}$ with $c_{r} \neq 0$ such that, for any $n \in \mathbb{N}$,

$$
w_{n+r}=c_{1} w_{n+r-1}+c_{2} w_{n+r-2}+\ldots+c_{r} w_{n} .
$$

Nishioka [16] verified for any integer $k \geq 2$ and any $\alpha$ that the set

$$
\left\{\varphi_{k}^{(l)}(\alpha) \mid l=0,1, \ldots\right\}
$$

is algebraically independent, using her criterion for algebraic independence in [14]. Considering the operator $X(d / d X)$, we see that the set

$$
\left\{\sum_{m=0}^{\infty} k^{l m} \alpha^{k^{m}} \mid l=0,1, \ldots\right\}
$$

is also algebraically independent. More generally, let $r$ be a positive integer and let $\boldsymbol{s}^{(1)}=\left(s_{1}(m)\right)_{m=0}^{\infty}, \ldots, \boldsymbol{s}^{(r)}=\left(s_{r}(m)\right)_{m=0}^{\infty}$ be linearly independent linear recurrences of integers. Using the same criterion in [14] as above, Nishioka [16] showed for any integer $k \geq 2$ and any $\alpha$ that the numbers

$$
\sum_{m=0}^{\infty} s_{i}(m) \alpha^{k^{m}} \quad(i=1, \ldots, r)
$$

are algebraically independent.

Tanaka [21] studied necessary and sufficient conditions for the algebraic independence of the values of $\sum_{m=0}^{\infty} X^{v_{m}}$ and their derivatives, where $\boldsymbol{v}=\left(v_{m}\right)_{m=0}^{\infty}$ are distinct linear recurrences satisfying certain assumptions. For instance, let $F_{m}(m=$ $0,1, \ldots)$ be the Fibonacci sequence defined by $F_{0}=0, F_{1}=1$, and $F_{m+2}=F_{m+1}+F_{m}$ for any $m=0,1, \ldots$. Put

$$
\varphi_{F}(X):=\sum_{m=0}^{\infty} X^{F_{m}}
$$


Then, for any $\alpha$, the set

$$
\left\{\varphi_{F}^{(l)}(\alpha) \mid l=0,1, \ldots\right\}
$$

is algebraically independent.

\section{Main results}

Let $\beta$ be a Pisot or Salem number and $\boldsymbol{t}=\left(t_{n}\right)_{n=0}^{\infty}$ a sequence of nonnegative integers such that $t_{n} \neq 0$ for infinitely many $n$ 's. In this section we study criteria for the algebraic independence of $f\left(\boldsymbol{t} ; \beta^{-1}\right)$ in the case where $f(\boldsymbol{t} ; X)$ is not generally a lacunary series. Note that we do not use functional equations for the criteria.

Let $\mathcal{A}$ be a nonempty subset of $\mathbb{N}$ and $k \in \mathbb{N}$. Then set

$$
k \mathcal{A}:=\left\{\begin{array}{cc}
\{0\} & (\text { if } k=0), \\
\left\{s_{1}+\ldots+s_{k} \mid s_{1}, \ldots, s_{k} \in \mathcal{A}\right\} & (\text { if } k \geq 1) .
\end{array}\right.
$$

Recall that $\lambda(\mathcal{A} ; R)$ is defined by (1.3). Moreover, $\left(\sigma_{1, \nu}(m)\right)_{m=1}^{\infty}$ for a positive real number $\nu$ and $\left(\sigma_{2}(m)\right)_{m=3}^{\infty}$ are defined in Section 1 .

We first consider the case where $\boldsymbol{t}=\left(t_{n}\right)_{n=0}^{\infty}$ is bounded. Using criteria for algebraic independence in [9], we obtain the following:

Theorem 3.1. ([9]) Let $\beta$ be a Pisot or Salem number.

(1) The continuum set

$$
\left\{\sum_{m=1}^{\infty} \beta^{-\left\lfloor\sigma_{1, \nu}(m)\right\rfloor} \mid \nu \in \mathbb{R}, \nu \geq 1\right\}
$$

is algebraically independent.

(2) Let $\nu$ and $\eta$ be distinct positive real numbers. Then the two numbers

$$
\sum_{m=1}^{\infty} \beta^{-\left\lfloor\sigma_{1, \nu}(m)\right\rfloor} \quad \text { and } \quad \sum_{m=1}^{\infty} \beta^{-\left\lfloor\sigma_{1, \eta}(m)\right\rfloor}
$$

are algebraically independent.

(3) Let $\nu$ be a positive real number. Then the two numbers

$$
\sum_{m=1}^{\infty} \beta^{-\left\lfloor\sigma_{1, \nu}(m)\right\rfloor} \text { and } \sum_{m=3}^{\infty} \beta^{-\left\lfloor\sigma_{2}(m)\right\rfloor}
$$

are algebraically independent. 
Note that if $\beta=b$ is an integer greater than 1 , then the first and second statements of Theorem 3.1 were obtained in [8].

In what follows, we introduce our main results. We now give a new criterion for algebraic independence applicable to some special cases, where $\boldsymbol{t}=\left(t_{n}\right)_{n=0}^{\infty}$ is unbounded.

Theorem 3.2. Let $\mathcal{A}$ be a set of nonnegative integers satisfying the following two assumptions:

1. There exists a positive constant $C_{1}>1$ such that

$$
\left[R, C_{1} R\right) \cap \mathcal{A} \neq \varnothing
$$

for any sufficiently large $R$.

2. For an arbitrary positive $\varepsilon$,

$$
\liminf _{R \rightarrow \infty} \frac{\lambda(\mathcal{A} ; R)}{R^{\varepsilon}}=0 .
$$

Let $\boldsymbol{t}^{(1)}=\left(t_{1}(n)\right)_{n=0}^{\infty}, \ldots, \boldsymbol{t}^{(r)}=\left(t_{r}(n)\right)_{n=0}^{\infty}$ be sequences of nonnegative integers with

$$
\mathcal{S}\left(\boldsymbol{t}^{(1)}\right)=\ldots=\mathcal{S}\left(\boldsymbol{t}^{(r)}\right)=\mathcal{A}
$$

satisfying the following two assumptions:

1. There exists a constant $0<C_{2}<1$ such that, for each $i=1, \ldots, r$,

$$
\log ^{+} t_{i}(n)=o\left(n^{1-C_{2}}\right)
$$

as $n$ tends to infinity, where $\log ^{+} x=\log \max \{1, x\}$ for a real number $x$.

2. Let $M$ be an arbitrary positive real number. Then, for $i=2, \ldots, r$,

$$
\lim _{n \in \mathcal{A}, n \rightarrow \infty} \frac{t_{i}(n)}{t_{i-1}(n)^{M}}=\infty .
$$

Let $\beta$ be a Pisot or Salem number. Then the numbers $f\left(\boldsymbol{t}^{(1)} ; \beta^{-1}\right), \ldots, f\left(\boldsymbol{t}^{(r)} ; \beta^{-1}\right)$ are algebraically independent.

Theorem 3.2 implies that if a set $\mathcal{A}$ of nonnegative integers satisfying (3.1) and (3.2) is given, then we can deduce examples of algebraically independent real numbers, changing sequences $\boldsymbol{t}^{(1)}, \ldots, \boldsymbol{t}^{(r)}$ of coefficients. Theorem 3.2 is applicable even if $\boldsymbol{t}^{(1)}, \ldots, \boldsymbol{t}^{(r)}$ are not linear recurrences.

We give examples of coefficients for a fixed infinite set $\mathcal{A}$ of nonnegative integers. Let $\boldsymbol{t}=\left(t_{n}\right)_{n=0}^{\infty}$ be a sequence of integers. Put

$$
f_{\mathcal{A}}(\boldsymbol{t} ; X)=\sum_{n \in \mathcal{A}} t_{n} X^{n} .
$$


Let $\Theta$ be the subset of $\mathbb{R}^{2}$ defined by

$$
\Theta:=\left\{(\mu, \nu) \in \mathbb{R}^{2} \mid 0<\mu<1\right\} \cup\left\{(0, \nu) \in \mathbb{R}^{2} \mid \nu \geq 0\right\} .
$$

Moreover, for any $(\mu, \nu) \in \Theta$, we define the sequence $\boldsymbol{r}_{\mu, \nu}=\left(r_{\mu, \nu}(n)\right)_{n=0}^{\infty}$ by

$$
r_{\mu, \nu}(n):=\left\lfloor\exp \left((n+2)^{\mu}(\log (n+2))^{\nu}\right)\right\rfloor .
$$

Corollary 3.3. Let $\mathcal{A}$ be a subset of $\mathbb{N}$ satisfying (3.1) and (3.2). Then, for any Pisot or Salem number $\beta$, the continuum set

$$
\mathcal{G}(\mathcal{A}):=\left\{f_{\mathcal{A}}\left(\boldsymbol{r}_{\mu, \nu} ; \beta^{-1}\right) \mid(\mu, \nu) \in \Theta\right\}
$$

is algebraically independent.

Corollary 3.3 is easily seen by Theorem 3.2. In fact, any element $f_{\mathcal{A}}\left(\boldsymbol{r}_{\mu, \nu} ; \beta^{-1}\right)$ of $\mathcal{G}(\mathcal{A})$ satisfies $(3.4)$ by $\mu<1$. Moreover, let $f_{\mathcal{A}}\left(\boldsymbol{r}_{\mu, \nu} ; \beta^{-1}\right)$ and $f_{\mathcal{A}}\left(\boldsymbol{r}_{\mu^{\prime}, \nu^{\prime}} ; \beta^{-1}\right)$ be any distinct two elements of $\mathcal{G}(\mathcal{A})$. Suppose that $\mu>\mu^{\prime}$, or $\mu=\mu^{\prime}$ and $\nu>\nu^{\prime}$. Then we see for any positive real number $M$ that

$$
\lim _{n \rightarrow \infty} \frac{r_{\mu, \nu}(n)}{r_{\mu^{\prime}, \nu^{\prime}}(n)^{M}}=\lim _{n \rightarrow \infty} \frac{\exp \left((n+2)^{\mu}(\log (n+2))^{\nu}\right)}{\exp \left(M(n+2)^{\mu^{\prime}}(\log (n+2))^{\nu^{\prime}}\right)}=\infty,
$$

which implies that (3.5) is also fulfilled.

Various sets $\mathcal{A}$ of nonnegative integers satisfy (3.1) and (3.2). Consider the case where $\mathcal{A}$ is denoted as

$$
\mathcal{A}=\left\{v_{m} \mid m \geq m_{0}\right\}
$$

where $m_{0}$ is a nonnegative integer and $\left(v_{m}\right)_{m=m_{0}}^{\infty}$ is an ultimately increasing sequence of nonnegative integers. We see that if $\left(v_{m}\right)_{m=m_{0}}^{\infty}$ satisfies (1.5) and

$$
\limsup _{m \rightarrow \infty} \frac{v_{m+1}}{v_{m}}<\infty
$$

then the set $\mathcal{A}$ defined by (3.6) fulfills (3.1) and (3.2). In fact, let $\varepsilon$ be an arbitrary positive real number. Using (1.5) with $A=1 / \varepsilon$, we see for any positive real number $y$ that there exist infinitely many positive integers $m$ with $v_{m}>y^{1 / \varepsilon} m^{1 / \varepsilon}$. Thus, we obtain that

$$
\frac{\lambda\left(\mathcal{A} ; y^{1 / \varepsilon} m^{1 / \varepsilon}\right)}{\left(y^{1 / \varepsilon} m^{1 / \varepsilon}\right)^{\varepsilon}}=\frac{\operatorname{Card}\left(\left[0, y^{1 / \varepsilon} m^{1 / \varepsilon}\right) \cap \mathcal{A}\right)}{y m} \leq \frac{1}{y}
$$

and that

$$
\liminf _{R \rightarrow \infty} \frac{\lambda(\mathcal{A} ; R)}{R^{\varepsilon}} \leq \frac{1}{y},
$$

which implies (3.2) because $y$ is an arbitrary positive real number. 
Recall that $v_{m}:=\left\lfloor\sigma_{1, \nu}(m)\right\rfloor(m \geq 1)$ satisfies (1.5) and (1.4) for any positive real number $\nu$. Thus, $\mathcal{A}_{1}:=\left\{\sigma_{1, \nu}(m) \mid m \geq 1\right\}$ satisfies (3.1) and (3.2). Hence, $\mathcal{A}_{1}$ is applicable to Theorem 3.2 or Corollary 3.3. In particular, Corollary 3.3 implies that

$$
\mathcal{G}\left(\mathcal{A}_{1}\right):=\left\{f_{\mathcal{A}_{1}}\left(\boldsymbol{r}_{\mu, \nu} ; \beta^{-1}\right) \mid(\mu, \nu) \in \Theta\right\}
$$

is algebraically independent. Note for any nonnegative integer $n$ that $r_{0,0}(n)=2$. Thus, we see

$$
\frac{1}{2} f_{\mathcal{A}_{1}}\left(\boldsymbol{r}_{0,0} ; \beta^{-1}\right)-\sum_{m=1}^{\infty} \beta^{-\left\lfloor\sigma_{1, \nu}(m)\right\rfloor} \in \mathbb{Q}(\beta)
$$

because $\left(\left\lfloor\sigma_{1, \nu}(m)\right\rfloor\right)_{m=1}^{\infty}$ is ultimately increasing. Therefore, we deduce the algebraic independence of real numbers including the value

$$
\sum_{m=1}^{\infty} \beta^{-\left\lfloor\sigma_{1, \nu}(m)\right\rfloor}
$$

Similarly, $\left\{\sigma_{2}(m) \mid m \geq 3\right\}$ also fulfills (3.1) and (3.2).

Moreover, we note that if $\left(v_{m}\right)_{m=m_{0}}^{\infty}$ satisfies

$$
1<\liminf _{m \rightarrow \infty} \frac{v_{m+1}}{v_{m}} \leq \limsup _{m \rightarrow \infty} \frac{v_{m+1}}{v_{m}}<\infty,
$$

then (1.5) and (3.7) are satisfied. In particular, the set $\left\{\left\lfloor w \eta^{m}\right\rfloor \mid m \geq 0\right\}$ for real numbers $w>0, \eta>1$, and the set $\left\{F_{m} \mid m \geq 0\right\}$ for the Fibonacci sequence $\left(F_{m}\right)_{m=0}^{\infty}$ fulfill (3.1) and (3.2).

In the last of this section we also deduce the algebraic independence of certain two numbers related to the derivatives of functions as follows:

Corollary 3.4. Let $\mathcal{A}$ be a subset of $\mathbb{N}$ satisfying (3.1) and (3.2). Let $\boldsymbol{t}=$ $(t(n))_{n=0}^{\infty}$ be a bounded sequence of positive integers. Let $\beta$ be a Pisot or Salem number. Then, for any positive integer $l$, the numbers $f_{\mathcal{A}}\left(\boldsymbol{t} ; \beta^{-1}\right)$ and $f_{\mathcal{A}}^{(l)}\left(\boldsymbol{t} ; \beta^{-1}\right)$ are algebraically independent.

Proof. Let $\gamma_{1}:=f_{\mathcal{A}}\left(\boldsymbol{t} ; \beta^{-1}\right)$ and $\gamma_{2}:=\beta^{-l} f_{\mathcal{A}}^{(l)}\left(\boldsymbol{t} ; \beta^{-1}\right)+\sum_{n<l, n \in \mathcal{A}} \beta^{-n}$, where

$$
\begin{aligned}
\gamma_{2} & =\sum_{n=l}^{\infty} n(n-1) \ldots(n-l+1) t_{n} \beta^{-n}+\sum_{n<l, n \in \mathbb{A}} \beta^{-n} \\
& =: \sum_{n=0}^{\infty} t_{2}(n) \beta^{-n} .
\end{aligned}
$$

Then two sequences $\boldsymbol{t}^{(1)}:=(t(n))_{n=0}^{\infty}$ and $\boldsymbol{t}^{(2)}:=\left(t_{2}(n)\right)_{n=0}^{\infty}$ satisfy (3.3), (3.4), and (3.5) because $\boldsymbol{t}^{(1)}$ is bounded. Thus, we obtain from Theorem 3.2 that $\gamma_{1}$ and $\gamma_{2}$ are algebraically independent, which implies the corollary. 


\section{Proof of Theorem 3.2}

We introduce notation. Let $k$ be a positive integer and $\boldsymbol{m}=\left(m_{1}, \ldots, m_{k}\right) \in \mathbb{N}^{k}$, $\underline{X}:=\left(X_{1}, \ldots, X_{k}\right)$. Put

$$
|\boldsymbol{m}|:=m_{1}+\ldots+m_{k} \quad \text { and } \quad \underline{X}^{\boldsymbol{m}}:=X_{1}^{m_{1}} \ldots X_{k}^{m_{k}} .
$$

For convenience, if $k=0$, then set

$$
|\boldsymbol{m}|:=0 \quad \text { and } \quad \underline{X}^{\boldsymbol{m}}:=1 \text {. }
$$

We write by $\succ_{\text {grl }}$ the graded reverse lexicographical order on $\mathbb{N}^{r}$ as follows: Let $\boldsymbol{k}=\left(k_{1}, \ldots, k_{r}\right), \boldsymbol{k}^{\prime}=\left(k_{1}^{\prime}, \ldots, k_{r}^{\prime}\right)$ be distinct elements of $\mathbb{N}^{r}$. Then $\boldsymbol{k} \succ_{\text {grl }} \boldsymbol{k}^{\prime}$ if and only if $|\boldsymbol{k}|>\left|\boldsymbol{k}^{\prime}\right|$, or $|\boldsymbol{k}|=\left|\boldsymbol{k}^{\prime}\right|$ and $k_{h}>k_{h}^{\prime}$, where

$$
h=\max \left\{1 \leq i \leq r \mid k_{i} \neq k_{i}^{\prime}\right\}
$$

In particular, we have

$$
(0, \ldots, 0,1) \succ_{\text {grl }}(0, \ldots, 0,1,0) \succ_{\text {grl }} \ldots \succ_{\text {grl }}(1,0, \ldots)
$$

Put $f_{i}(X):=f\left(\boldsymbol{t}^{(i)} ; X\right)$ and $\xi_{i}:=f_{i}\left(\beta^{-1}\right)$ for $i=1, \ldots, r$. Set $\mathbf{0}:=(0, \ldots, 0) \in \mathbb{N}^{r}$ and $\xi:=$ $\left(\xi_{1}, \ldots, \xi_{r}\right)$. In what follows, we show that $P(\underline{\xi}) \neq 0$ for any nonzero polynomial $P(\underline{X})$ whose coefficients are integers. Let $D$ be the total degree of $P(\underline{X})$. Throughout the proof of Theorem 3.2, we consider the set $(D-1) \mathcal{B}$, where $\mathcal{B}$ is defined later. However, $(D-1) \mathcal{B}$ is defined only if $D-1 \geq 1$. For simplicity, we may assume that $D \geq 2$. In fact, if $D=1$, then it suffices to show that the polynomial $X_{1} P(\underline{X})$ does not vanish at $\underline{X}=\underline{\xi}$. Let

$$
P(\underline{X})=: A_{0}+\sum_{k \in \Lambda} A_{k} \underline{X}^{k}
$$

where $\Lambda$ is a nonempty subset of $\mathbb{N}^{r} \backslash\{\mathbf{0}\}, A_{\mathbf{0}} \in \mathbb{Z}$, and $A_{\boldsymbol{k}} \in \mathbb{Z} \backslash\{0\}$ for any $\boldsymbol{k} \in \Lambda$. We write by $\boldsymbol{g}=\left(g_{1}, \ldots, g_{r}\right)$ the maximal element of $\Lambda$ with respect to $\succ_{\text {grl }}$. Without loss of generality, we may assume that $A_{\boldsymbol{g}}>0$. Set

$$
\Lambda_{1}:=\{\boldsymbol{k} \in \Lambda \backslash\{\boldsymbol{g}\}|| \boldsymbol{k} \mid=D\} \quad \text { and } \quad \Lambda_{2}:=\{\boldsymbol{k} \in \Lambda \backslash\{\boldsymbol{g}\}|| \boldsymbol{k} \mid<D\} .
$$

Then we have

$$
\Lambda=\{\boldsymbol{g}\} \cup \Lambda_{1} \cup \Lambda_{2}
$$


In what follows, the implied constants in the symbols $\ll$ and $\gg$ and $C_{3}, C_{4}, \ldots$ are positive constants depending only on $f_{1}(X), \ldots, f_{r}(X), \beta$, and $P(\underline{X})$. Set

$$
C_{3}:=\max \left\{1, \frac{2(D !)}{A_{\boldsymbol{g}}}\left(1+\sum_{\boldsymbol{k} \in \Lambda_{1}}\left|A_{\boldsymbol{k}}\right|\right)\right\} .
$$

By (3.5), there exists a positive integer $C_{4}$ such that if $n$ is an element of $\mathcal{A}$ with $n \geq C_{4}$, then

$$
t_{u}(n)>C_{3}^{D} t_{v}(n)^{D}
$$

for any integers $u, v$ with $1 \leq v<u \leq r$. For simplicity, we consider $\eta_{i}=\varphi_{i}\left(\beta^{-1}\right)$ instead of $\xi_{i}$ for $i=1, \ldots, r$, where

$$
\varphi_{i}(X)=\sum_{n=0}^{\infty} s_{i}(n) X^{n}:=1+\sum_{n=C_{4}}^{\infty} t_{i}(n) X^{n} .
$$

Then we see $\beta^{C_{4}}\left(\xi_{i}-\eta_{i}\right) \in \mathbb{Z}[\beta]$. Observe for each $\boldsymbol{k}=\left(k_{1}, \ldots, k_{r}\right) \in \Lambda$ that

$$
\beta^{C_{4} D} A_{\boldsymbol{k}} \underline{\xi}^{\boldsymbol{k}}=\beta^{C_{4}(D-|\boldsymbol{k}|)} A_{\boldsymbol{k}} \prod_{i=1}^{r}\left(\beta^{C_{4}}\left(\xi_{i}-\eta_{i}\right)+\beta^{C_{4}} \eta_{i}\right)^{k_{i}}
$$

is a polynomial of $\eta=\left(\eta_{1}, \ldots, \eta_{r}\right)$ whose coefficients are elements of $\mathbb{Z}[\beta]$. Expanding $\beta^{C_{4} D} P(\underline{\xi})$ by the relation above, we see that

$$
\beta^{C_{4} D} P(\underline{\xi})=: Q(\underline{\eta})=B_{\mathbf{0}}+\sum_{\boldsymbol{k} \in \Gamma} B_{\boldsymbol{k}} \underline{\eta}^{\boldsymbol{k}}
$$

where $Q(\underline{X}) \in \mathbb{Z}[\beta]\left[X_{1}, \ldots, X_{r}\right]$ has total degree $D(\geq 2), \Gamma$ is a nonempty subset of $\mathbb{N}^{r} \backslash\{\mathbf{0}\}, B_{\mathbf{0}} \in \mathbb{Z}[\beta]$, and $B_{\boldsymbol{k}} \in \mathbb{Z}[\beta] \backslash\{0\}$ for any $\boldsymbol{k} \in \Gamma$. By the definition of $Q(\underline{X})$, the maximal element of $\Gamma$ with respect to $\succ_{\text {grl }}$ is $\boldsymbol{g}$. Similarly, putting

$$
\Gamma_{1}:=\{\boldsymbol{k} \in \Gamma \backslash\{\boldsymbol{g}\}|| \boldsymbol{k} \mid=D\} \quad \text { and } \quad \Gamma_{2}:=\{\boldsymbol{k} \in \Gamma \backslash\{\boldsymbol{g}\}|| \boldsymbol{k} \mid<D\}
$$

we get $\Gamma_{1}=\Lambda_{1}$ and

$$
B_{k}=\beta^{C_{4} D} A_{k}
$$

for any $\boldsymbol{k} \in\{\boldsymbol{g}\} \cup \Gamma_{1}$. In particular, we have $B_{\boldsymbol{g}}>0$. Note that

$$
\Gamma=\{\boldsymbol{g}\} \cup \Gamma_{1} \cup \Gamma_{2} .
$$


We check that the power series $\varphi_{1}(X), \ldots, \varphi_{r}(X)$ with nonnegative integral coefficients satisfy the assumptions of Theorem 3.2. In particular, we get

$$
\begin{aligned}
\mathcal{S}\left(\varphi_{1}\right) & =\ldots=\mathcal{S}\left(\varphi_{r}\right) \\
& =\{0\} \cup\left(\left[C_{4}, \infty\right) \cap \mathcal{A}\right)=: \mathcal{B}
\end{aligned}
$$

by $(3.3),(4.2)$, and so

$$
\left[R, C_{1} R\right) \cap \mathcal{B} \neq \varnothing
$$

for any sufficiently large $R$ by (3.1). Moreover, putting

$$
\lambda(\mathcal{B} ; R)=: \lambda(R) \quad \text { and } \quad \mathcal{C}:=C_{2} / 2
$$

for simplicity, we see for an arbitrary positive real $\varepsilon$ that

$$
\liminf _{R \rightarrow \infty} \frac{\lambda(R)}{R^{\varepsilon}}=0
$$

by (3.2). By considering the case of $\varepsilon=\mathcal{C} /(2 D-1)$, there exists an infinite set $\mathcal{F}$ of nonnegative integers such that

$$
4^{-1 /(2 D-1)}\left(1+C_{1}\right)^{-1 /(2 D-1)}>\frac{\lambda(N)}{N^{\mathcal{C} /(2 D-1)}}
$$

for any $N \in \mathcal{F}$. Thus, we get for any $N \in \mathcal{F}$ that

$$
N^{\mathcal{C}}>4\left(1+C_{1}\right) \lambda(N)^{2 D-1}
$$

We see by (3.4) that

$$
\log ^{+} s_{i}(n)=o\left(n^{1-2 \mathcal{C}}\right)
$$

as $n$ tends to infinity, where $0<2 \mathcal{C}<1$. Moreover, using (4.1) and (4.2), we get

$$
s_{u}(n)>C_{3}^{D} s_{v}(n)^{D}
$$

for any integers $u, v$ with $1 \leq v<u \leq r$ and any $n \in \mathcal{B} \backslash\{0\}$.

We now calculate $\eta^{\boldsymbol{k}}$ for $\boldsymbol{k} \in \Gamma$. Let $k$ be a positive integer and $\boldsymbol{m}=\left(m_{1}, \ldots, m_{k}\right) \in$ $\mathbb{N}^{k}$. For $i=1, \ldots, r$, put

$$
s_{i}(\boldsymbol{m}):=s_{i}\left(m_{1}\right) \ldots s_{i}\left(m_{k}\right) .
$$


For convenience, if $k=0$, then we set $s_{i}(\boldsymbol{m}):=1$. For a positive integer $m$, we denote by $\mathcal{B}^{m}$ the $m$ th Cartesian power of $\mathcal{B}$. Moreover, put $\mathcal{B}^{0}:=\{0\}$. For any $\underline{\eta}^{\boldsymbol{k}}$ with $\boldsymbol{k}=\left(k_{1}, \ldots, k_{r}\right) \in \Gamma$, we see

$$
\begin{aligned}
\underline{\eta}^{\boldsymbol{k}} & =\prod_{i=1}^{r}\left(\sum_{m \in \mathcal{B}} s_{i}(m) \beta^{-m}\right)^{k_{i}}=\prod_{i=1}^{r}\left(\sum_{\boldsymbol{m}_{i} \in \mathcal{B}^{k_{i}}} s_{i}\left(\boldsymbol{m}_{i}\right) \beta^{-\left|\boldsymbol{m}_{i}\right|}\right) \\
& =\sum_{\boldsymbol{m}_{1} \in \mathcal{B}^{k_{1}, \ldots, \boldsymbol{m}_{r} \in \mathcal{B}^{k_{r}}}} s_{1}\left(\boldsymbol{m}_{1}\right) \ldots s_{r}\left(\boldsymbol{m}_{r}\right) \beta^{-\left(\left|\boldsymbol{m}_{1}\right|+\ldots+\left|\boldsymbol{m}_{r}\right|\right)} \\
& =: \sum_{m=0}^{\infty} \beta^{-m} \rho(\boldsymbol{k} ; m),
\end{aligned}
$$

where

$$
\rho(\boldsymbol{k} ; m)=\sum_{\substack{\boldsymbol{m}_{1} \in \mathcal{B}^{k_{1}}, \ldots, \boldsymbol{m}_{r} \in \mathcal{B}^{k_{r}} \\\left|\boldsymbol{m}_{1}\right|+\ldots+\left|\boldsymbol{m}_{r}\right|=m}} s_{1}\left(\boldsymbol{m}_{1}\right) \ldots s_{r}\left(\boldsymbol{m}_{r}\right)
$$

is a nonnegative integer. Substituting (4.10) into (4.3), we get

$$
\begin{aligned}
\beta^{C_{4} D} P(\underline{\xi}) & =Q(\underline{\eta})=B_{\mathbf{0}}+\sum_{\boldsymbol{k} \in \Gamma} B_{\boldsymbol{k}} \underline{\eta}^{\boldsymbol{k}} \\
& =B_{\mathbf{0}}+\sum_{\boldsymbol{k} \in \Gamma} B_{\boldsymbol{k}} \sum_{m=0}^{\infty} \beta^{-m} \rho(\boldsymbol{k} ; m) .
\end{aligned}
$$

Let $R$ be a nonnegative integer. Then

$$
\beta^{R+C_{4} D} P(\underline{\xi})=B_{\mathbf{0}} \beta^{R}+\sum_{\boldsymbol{k} \in \Gamma} B_{\boldsymbol{k}} \sum_{m=-R}^{\infty} \beta^{-m} \rho(\boldsymbol{k} ; m+R) .
$$

Put

$$
\begin{aligned}
& Y_{R}:=\sum_{\boldsymbol{k} \in \Gamma} B_{\boldsymbol{k}} \sum_{m=1}^{\infty} \beta^{-m} \rho(\boldsymbol{k} ; m+R), \\
& Z_{R}:=B_{\mathbf{0}} \beta^{R}+\sum_{\boldsymbol{k} \in \Gamma} B_{\boldsymbol{k}} \sum_{m=-R}^{0} \beta^{-m} \rho(\boldsymbol{k} ; m+R) .
\end{aligned}
$$

Then we have

$$
\beta^{R+C_{4} D} P(\underline{\xi})=Y_{R}+Z_{R}
$$

Note that $Z_{R} \in \mathbb{Z}[\beta]$ because $B_{\boldsymbol{k}} \in \mathbb{Z}[\beta]$ for any $\boldsymbol{k} \in\{\mathbf{0}\} \cup \Gamma$. 
We now introduce a sketch of the proof of Theorem 3.2. We first show for any nonnegative integer $R$ that

$$
Z_{R}=0 \quad \text { or } \quad\left|Z_{R}\right| \geq C_{5} \beta^{-R^{1-2 \mathcal{C}}}
$$

in Lemma 4.2. Next, we show that there exists a positive integer $R$ satisfying

$$
0<Y_{R}<C_{5} \beta^{-R^{1-2 C}}
$$

in Lemma 4.6. Therefore, (4.11) implies that

$$
P(\underline{\xi}) \neq 0 \text {. }
$$

Since $P(\underline{X})$ is any non-constant polynomial whose coefficients are elements in $\mathbb{Z}[\beta]$, we deduce that $\xi_{1}, \ldots, \xi_{r}$ are algebraically independent.

We now give upper bounds for $\rho(\boldsymbol{k} ; m)$.

Lemma 4.1. For any $\boldsymbol{k} \in \Gamma$, we have

$$
\log ^{+} \rho(\boldsymbol{k} ; m)=o\left(m^{1-2 \mathcal{C}}\right) .
$$

In particular, there exists a positive constant $C_{6}$ satisfying the following: for any $\boldsymbol{k} \in \Gamma$ and $m \in \mathbb{N}$,

$$
\rho(\boldsymbol{k} ; m) \leq C_{6} \beta^{m^{1-2 C}} .
$$

Proof. Let $\boldsymbol{k} \in \Gamma$. Using (4.9) and the definition of $\rho(\boldsymbol{k} ; m)$, we get

$$
\begin{aligned}
\rho(\boldsymbol{k} ; m) & \leq(m+1)^{k_{1}+\ldots+k_{r}}\left(\max _{\substack{1 \leq i \leq r \\
0 \leq n \leq m}} s_{i}(n)\right)^{k_{1}+\ldots+k_{r}} \\
& \leq(m+1)^{D}\left(\max _{\substack{1 \leq i \leq r \\
0 \leq n \leq m}} s_{i}(n)\right)^{D} .
\end{aligned}
$$

Taking the logarithm of the inequality above, we obtain, by (4.7),

$$
\log ^{+} \rho(\boldsymbol{k} ; m)=o\left(m^{1-2 \mathcal{C}}\right)
$$

as $m$ tends to infinity. Moreover, the second assertion also holds because $\Gamma$ is a finite set.

Lemma 4.2. There exists a positive constant $C_{5}$ satisfying the following: For any nonnegative integer $R$, we have

$$
Z_{R}=0 \quad \text { or } \quad\left|Z_{R}\right| \geq C_{5} \beta^{-R^{1-2 \mathcal{C}}} .
$$


Proof. Put $d:=\operatorname{deg} \beta$. Let $\pi_{1}, \ldots, \pi_{d}$ be the conjugate embeddings of $\mathbb{Q}(\beta)$ into $\mathbb{C}$, where $\pi_{1}(\gamma)=\gamma$ for any $\gamma \in \mathbb{Q}(\beta)$. Set

$$
C_{7}:=\max \left\{\left|\pi_{i}\left(B_{\boldsymbol{k}}\right)\right| \mid i=1, \ldots, d, \boldsymbol{k} \in\{\mathbf{0}\} \cup \Gamma\right\} .
$$

Setting $\pi_{i}(\beta)=: \beta_{i}$ for $i=2, \ldots, d$, we have

$$
\pi_{i}\left(Z_{R}\right)=\pi_{i}\left(B_{\mathbf{0}}\right) \beta_{i}^{R}+\sum_{\boldsymbol{k} \in \Gamma} \pi_{i}\left(B_{\boldsymbol{k}}\right) \sum_{m=0}^{R} \beta_{i}^{m} \rho(\boldsymbol{k} ;-m+R) .
$$

Recall that $\left|\beta_{i}\right| \leq 1$ because $\beta$ is a Pisot or Salem number. Thus,

$$
\left|\pi_{i}\left(Z_{R}\right)\right| \leq C_{7}+\sum_{\boldsymbol{k} \in \Gamma} C_{7}(R+1) \max _{0 \leq m \leq R} \rho(\boldsymbol{k} ; m) .
$$

Using Lemma 4.1, we get, for $i=2, \ldots, d$,

$$
\log ^{+}\left|\pi_{i}\left(Z_{R}\right)\right|=o\left(R^{1-2 \mathcal{C}}\right)
$$

as $R$ tends to infinity. Hence, we see for any sufficiently large $R$ that

$$
\sum_{i=2}^{d} \log ^{+}\left|\pi_{i}\left(Z_{R}\right)\right| \leq R^{1-2 \mathcal{C}} \log \beta
$$

and so

$$
\prod_{i=2}^{d}\left|\pi_{i}\left(Z_{R}\right)\right| \leq \beta^{R^{1-2 \mathcal{C}}} .
$$

Assume that $Z_{R} \neq 0$. Since $Z_{R}$ is an algebraic integer, we obtain

$$
1 \leq\left|Z_{R}\right| \prod_{i=2}^{d}\left|\pi_{i}\left(Z_{R}\right)\right|
$$

Therefore, we deduce for any sufficiently large $R$ that

$$
\left|Z_{R}\right| \geq \beta^{-R^{1-2 \mathcal{C}}}
$$

In what follows, we consider lower bounds for $Y_{R}$.

Lemma 4.3. Let $\boldsymbol{k} \in \Gamma$ and let $m$ be a nonnegative integer. Then $\rho(\boldsymbol{k} ; m)$ is positive if and only if $m \in|\boldsymbol{k}| \mathcal{B}$. 
Proof. We observe that $\rho(\boldsymbol{k} ; m)$ is positive if and only if there exist $\boldsymbol{m}_{1} \in \mathcal{B}^{k_{1}}, \ldots$, $\boldsymbol{m}_{r} \in \mathcal{B}^{k_{r}}$ such that $m=\sum_{i=1}^{r}\left|\boldsymbol{m}_{i}\right|$. Thus, $\rho(\boldsymbol{k} ; m)$ is positive if and only if there exists an $\boldsymbol{n} \in \mathcal{B}^{k_{1}} \times \ldots \times \mathcal{B}^{k_{r}}$ such that $m=|\boldsymbol{n}|$, that is, $|\boldsymbol{n}| \in|\boldsymbol{k}| \mathcal{B}$ because $\mathcal{B}^{k_{i}}=\{0\}$ for any $1 \leq i \leq r$ with $k_{i}=0$.

Since $0 \in \mathcal{B}$, we get

$$
\mathcal{B} \subset 2 \mathcal{B} \subset \ldots \subset D \mathcal{B}
$$

Let $\boldsymbol{k} \in \Gamma$ and $\boldsymbol{n}=(n(1), \ldots, n(|\boldsymbol{k}|)) \in \mathbb{N}^{|\boldsymbol{k}|}$. We divide $\boldsymbol{n}$ into $r$ parts as follows:

$$
\begin{aligned}
\boldsymbol{n} & =(\underbrace{n(1), \ldots, n\left(k_{1}\right)}, \underbrace{n\left(k_{1}+\ldots+k_{r-1}+1\right), \ldots, n\left(k_{1}+\ldots+k_{r-1}+k_{r}\right)}) \\
& =:(\boldsymbol{n}(\boldsymbol{k}, 1), \boldsymbol{n}(\boldsymbol{k}, 2), \ldots, \boldsymbol{n}(\boldsymbol{k}, r)),
\end{aligned}
$$

where

$$
\boldsymbol{n}(\boldsymbol{k}, i):=\left(n\left(k_{1}+\ldots+k_{i-1}+1\right), \ldots, n\left(k_{1}+\ldots+k_{i-1}+k_{i}\right)\right)
$$

for $i=1, \ldots, r$. Let

$$
s(\boldsymbol{k} ; \boldsymbol{n}):=\prod_{i=1}^{r} s_{i}(\boldsymbol{n}(\boldsymbol{k}, i)),
$$

where $s_{i}(\boldsymbol{n}(\boldsymbol{k}, i))$ is defined by (4.9).

Lemma 4.4. Let $m$ be any nonnegative integer with $m \notin(D-1) \mathcal{B}$. Then

$$
\sum_{\boldsymbol{k} \in \Gamma \backslash\{\boldsymbol{g}\}}\left|B_{\boldsymbol{k}}\right| \rho(\boldsymbol{k} ; m) \leq \frac{1}{2} B_{\boldsymbol{g}} \rho(\boldsymbol{g} ; m) .
$$

Proof. Without loss of generality, we may assume that $m \in D \mathcal{B}$. In fact, if $m \notin D \mathcal{B}$, then we see that both-hand sides of (4.16) are 0 by Lemma 4.3 and (4.13).

Using Lemma 4.3 , we see for any $\boldsymbol{k} \in \Gamma_{2}$ that $\rho(\boldsymbol{k} ; m)=0$ by $(4.13)$ and $m \notin$ $(D-1) \mathcal{B}$. Thus,

$$
\sum_{\boldsymbol{k} \in \Gamma \backslash\{\boldsymbol{g}\}}\left|B_{\boldsymbol{k}}\right| \rho(\boldsymbol{k} ; m)=\sum_{\boldsymbol{k} \in \Gamma_{1}}\left|B_{\boldsymbol{k}}\right| \rho(\boldsymbol{k} ; m) .
$$

Hence, we may assume for the proof of Lemma 4.4 that $\Gamma_{1}$ is not empty. In particular, we have $r \geq 2$. Note for any $\boldsymbol{k} \in\{\boldsymbol{g}\} \cup \Gamma_{1}$ that $\rho(\boldsymbol{k} ; m)>0$ by Lemma 4.3 and $|\boldsymbol{k}|=D$. 
Put

$$
\Xi:=\left\{\boldsymbol{n}=(n(1), \ldots, n(D)) \in \mathcal{B}^{D}|| \boldsymbol{n} \mid=m\right\}(\neq \varnothing) .
$$

We apply notation (4.14) and (4.15) to $\boldsymbol{k} \in\{\boldsymbol{g}\} \cup \Gamma_{1}$ and $\boldsymbol{n} \in \Xi \subset \mathbb{N}^{|\boldsymbol{k}|}$. Then we see for any $\boldsymbol{k} \in\{\boldsymbol{g}\} \cup \Gamma_{1}$ that

$$
\rho(\boldsymbol{k} ; m)=\sum_{\boldsymbol{n} \in \mathcal{B}^{D},|\boldsymbol{n}|=m} s_{1}(\boldsymbol{n}(\boldsymbol{k}, 1)) \ldots s_{r}(\boldsymbol{n}(\boldsymbol{k}, r))=\sum_{\boldsymbol{n} \in \Xi} s(\boldsymbol{k} ; \boldsymbol{n}) .
$$

We first show for any fixed $\boldsymbol{k}=\left(k_{1}, \ldots, k_{r}\right) \in \Gamma_{1}$ that

$$
\rho(\boldsymbol{k} ; m)<\frac{D !}{C_{3}} \rho(\boldsymbol{g} ; m) \text {. }
$$

Put

$$
l:=\max \left\{i \geq 1 \mid k_{i} \neq g_{i}\right\}
$$

Since $|\boldsymbol{k}|=|\boldsymbol{g}|=D$, we have $l \geq 2$ because there exists an integer $a$ with $a<l$ satisfying $g_{a}<k_{a}$. Moreover, setting

$$
\tau:=D-\left(k_{l}+k_{l+1}+\ldots+k_{r}\right)=k_{1}+k_{2}+\ldots+k_{l-1},
$$

we see that $\tau>0$.

Let $\boldsymbol{n}=(n(1), \ldots, n(D)) \in \Xi$. Take an integer $b$ with $1 \leq b \leq \tau$ satisfying

$$
s_{l}(n(b))=\max _{1 \leq j \leq \tau} s_{l}(n(j))
$$

There exists a permutation $\sigma$ of the set $\{1, \ldots, \tau\}$ such that

$$
\sigma(\tau)=b
$$

Let

$$
(p(1), \ldots, p(\tau)):=(n(\sigma(1)), \ldots, n(\sigma(\tau)))
$$

and

$$
\boldsymbol{p}:=(p(1), p(2), \ldots, p(\tau), n(\tau+1), n(\tau+2), \ldots, n(D)) .
$$

It is clear that $\boldsymbol{p} \in \Xi$ because $\boldsymbol{p}$ is obtained by a permutation of the components of $\boldsymbol{n}$. Note that $\boldsymbol{p}$ is determined by $\boldsymbol{k} \in \Gamma_{1}$ and $\boldsymbol{n} \in \Xi$. Recall that

$$
s(\boldsymbol{k} ; \boldsymbol{n})=\prod_{i=1}^{r} s_{i}(\boldsymbol{n}(\boldsymbol{k}, i)) \quad \text { and } \quad s(\boldsymbol{g} ; \boldsymbol{p})=\prod_{i=1}^{r} s_{i}(\boldsymbol{p}(\boldsymbol{g}, i)),
$$


where $\boldsymbol{n}(\boldsymbol{k}, i) \in \mathcal{B}^{k_{i}}$ and $\boldsymbol{p}(\boldsymbol{g}, i) \in \mathcal{B}^{g_{i}}$ for $i=1, \ldots, r$. By the definition of $\boldsymbol{p}$, the last $(D-\tau)$-th components of $\boldsymbol{n}$ and $\boldsymbol{p}$ coincide. Observing that $g_{i}=k_{i}$ for any $i \geq l+1$, we see

$$
\boldsymbol{n}(\boldsymbol{k}, i)=\boldsymbol{p}(\boldsymbol{g}, i)
$$

for each $i \geq l+1$ because $k_{l+1}+\ldots+k_{r} \leq D-r$ by the definition of $\tau$. Moreover, since $g_{l}>k_{l}$, we see that $\boldsymbol{n}(\boldsymbol{k}, l)$ and $\boldsymbol{p}(\boldsymbol{g}, l)$ are denoted as

$$
\begin{aligned}
\boldsymbol{n}(\boldsymbol{k}, l) & =\left(n(\tau+1), \ldots, n\left(\tau+k_{l}\right)\right), \\
\boldsymbol{p}(\boldsymbol{g}, l) & =\left(p(\tau-h+1), \ldots, p(\tau), n(\tau+1), \ldots, n\left(\tau+k_{l}\right)\right),
\end{aligned}
$$

respectively, where $h=g_{l}-k_{l}>0$. Thus, we have

$$
s_{l}(\boldsymbol{p}(\boldsymbol{g}, l))=s_{l}(\boldsymbol{n}(\boldsymbol{k}, l)) \prod_{j=1}^{h} s_{l}(p(\tau+1-j)) .
$$

Hence, combining (4.22) and (4.23), we obtain

$$
\begin{aligned}
s(\boldsymbol{g} ; \boldsymbol{p}) & =\prod_{i=1}^{r} s_{i}(\boldsymbol{p}(\boldsymbol{g}, i)) \geq s_{l}(\boldsymbol{p}(\boldsymbol{g}, l)) \prod_{i=l+1}^{r} s_{i}(\boldsymbol{p}(\boldsymbol{g}, i)) \\
& =s_{l}(\boldsymbol{n}(\boldsymbol{k}, l)) \prod_{j=1}^{h} s_{l}(p(\tau+1-j)) \prod_{i=l+1}^{r} s_{i}(\boldsymbol{n}(\boldsymbol{k}, i)) \\
& =\prod_{j=1}^{h} s_{l}(p(\tau+1-j)) \prod_{i=l}^{r} s_{i}(\boldsymbol{n}(\boldsymbol{k}, i)) \geq s_{l}(p(\tau)) \prod_{i=l}^{r} s_{i}(\boldsymbol{n}(\boldsymbol{k}, i)) .
\end{aligned}
$$

Note that $n(j)>0$ for any $1 \leq j \leq D$. In fact, if $n\left(j_{0}\right)=0$ for some $1 \leq j_{0} \leq D$, then

$$
m=|\boldsymbol{n}|=\sum_{\substack{1 \leq j \leq D \\ j \neq j_{0}}} n(j) \in(D-1) \mathcal{B},
$$

which contradicts the assumption of Lemma 4.4.

We now let $i, j$ be integers with $1 \leq i \leq l-1$ and $1 \leq j \leq \tau$. Applying (4.8) with $u=l, v=i$, and $n=n(j) \in \mathcal{B} \backslash\{0\}$, we see

$$
\begin{aligned}
C_{3} s_{i}(n(j)) & <s_{l}(n(j))^{1 / D} \leq s_{l}(n(b))^{1 / D} \\
& =s_{l}(p(\tau))^{1 / D}
\end{aligned}
$$

by $(4.20)$ and $p(\tau)=n(\sigma(\tau))=n(b)$. In particular, we get

$$
C_{3}^{\tau} \max _{\substack{1 \leq j \leq \tau \\ 1 \leq i \leq l-1}} s_{i}(n(j))^{\tau}<s_{l}(p(\tau))^{\tau / D} \leq s_{l}(p(\tau))
$$


by $1 \leq \tau \leq D$, and so

$$
\begin{aligned}
C_{3} \prod_{i=1}^{l-1} s_{i}(\boldsymbol{n}(\boldsymbol{k}, i)) & \leq C_{3} \prod_{i=1}^{l-1} \max _{1 \leq j \leq \tau} s_{i}(n(j))^{k_{i}} \\
& \leq C_{3}^{\tau} \max _{\substack{1 \leq j \leq \tau \\
1 \leq i \leq l-1}} s_{i}(n(j))^{\tau}<s_{l}(p(\tau))
\end{aligned}
$$

by $C_{3} \geq 1$. Combining (4.24) and (4.25), we obtain

$$
s(\boldsymbol{g} ; \boldsymbol{p})>C_{3} \prod_{i=1}^{l-1} s_{i}(\boldsymbol{n}(\boldsymbol{k}, i)) \prod_{i=l}^{r} s_{i}(\boldsymbol{n}(\boldsymbol{k}, i))=C_{3} s(\boldsymbol{k} ; \boldsymbol{n}) .
$$

Let $\boldsymbol{n}, \boldsymbol{n}^{\prime} \in \Xi$. We write $\boldsymbol{n} \sim \boldsymbol{n}^{\prime}$ if $\boldsymbol{n}$ is translated to $\boldsymbol{n}^{\prime}$ by a permutation of the components. Note that $\boldsymbol{p} \sim \boldsymbol{n}$ for any $\boldsymbol{n} \in \Xi$, where $\boldsymbol{p}$ is defined by (4.21). We write $\boldsymbol{p}=: \boldsymbol{p}(\boldsymbol{n})$ in order to emphasize that $\boldsymbol{p}$ depends on $\boldsymbol{n}$. We denote the set of equivalence classes of $\Xi$ with respect to $\sim$ by $\Xi / \sim$. Using (4.18) and (4.26), we obtain

$$
\begin{aligned}
\rho(\boldsymbol{k} ; m) & =\sum_{\boldsymbol{n} \in \Xi} s(\boldsymbol{k} ; \boldsymbol{n})=\sum_{\alpha \in \Xi / \sim} \sum_{\boldsymbol{n} \in \alpha} s(\boldsymbol{k} ; \boldsymbol{n}) \\
& <\frac{1}{C_{3}} \sum_{\alpha \in \Xi / \sim} \sum_{\boldsymbol{n} \in \alpha} s(\boldsymbol{g} ; \boldsymbol{p}(\boldsymbol{n})) .
\end{aligned}
$$

Observe for any $\alpha \in \Xi / \sim$ that

$$
\text { Card } \alpha \leq D !
$$

Moreover, if $\boldsymbol{n} \in \alpha$, then $\boldsymbol{p}(\boldsymbol{n}) \in \alpha$. Hence,

$$
\begin{aligned}
\rho(\boldsymbol{k} ; m) & <\frac{1}{C_{3}} \sum_{\alpha \in \Xi / \sim} D ! \max _{\boldsymbol{n} \in \alpha} s(\boldsymbol{g} ; \boldsymbol{n}) \\
& \leq \frac{D !}{C_{3}} \sum_{\alpha \in \Xi / \sim \boldsymbol{n} \in \alpha} \sum_{\alpha} s(\boldsymbol{g} ; \boldsymbol{n})=\frac{D !}{C_{3}} \sum_{\boldsymbol{n} \in \Xi} s(\boldsymbol{g} ; \boldsymbol{n})=\frac{D !}{C_{3}} \rho(\boldsymbol{g} ; m)
\end{aligned}
$$

by (4.18), which implies (4.19).

Recall that $\Gamma_{1}=\Lambda_{1}$ and $A_{g}>0$. Applying (4.17), (4.4), (4.19), the definition of $C_{3}$, we deduce that

$$
\begin{aligned}
\sum_{\boldsymbol{k} \in \Gamma \backslash\{\boldsymbol{g}\}}\left|B_{\boldsymbol{k}}\right| \rho(\boldsymbol{k} ; m) & =\sum_{\boldsymbol{k} \in \Lambda_{1}} \beta^{C_{4} D}\left|A_{\boldsymbol{k}}\right| \rho(\boldsymbol{k} ; m)<\sum_{\boldsymbol{k} \in \Lambda_{1}} \beta^{C_{4} D}\left|A_{\boldsymbol{k}}\right| \frac{D !}{C_{3}} \rho(\boldsymbol{g} ; m) \\
& =\beta^{C_{4} D} \rho(\boldsymbol{g} ; m) \cdot \frac{D !}{C_{3}} \sum_{\boldsymbol{k} \in \Lambda_{1}}\left|A_{\boldsymbol{k}}\right| \\
& \leq \beta^{C_{4} D} \rho(\boldsymbol{g} ; m) \cdot \frac{1}{2} A_{\boldsymbol{g}}=\frac{1}{2} B_{\boldsymbol{g}} \rho(\boldsymbol{g} ; m),
\end{aligned}
$$

which implies Lemma 4.4. 
Let $k$ be a positive integer. Then, for any positive integer $N$,

$$
\lambda(k \mathcal{B} ; N)=\operatorname{Card}(k \mathcal{B} \cap[0, N)) \leq \operatorname{Card}(\mathcal{B} \cap[0, N))^{k}=\lambda(N)^{k} .
$$

For any interval $I=[x, y) \subset \mathbb{R}$ with $x<y$, we denote its length by $|I|:=y-x$.

Let $N$ be a sufficiently large element of $\mathcal{F}$. Set

$$
[0, N) \cap(D-1) \mathcal{B}=:\left\{0=j_{1}<j_{2}<\ldots<j_{\varkappa}\right\},
$$

and $j_{1+\varkappa}:=N$. Recall that $D \geq 2$. Applying (4.27) with $k=D-1 \geq 1$, we see

$$
\varkappa \leq \lambda(N)^{D-1} \text {. }
$$

Observe that $j_{a} \in(D-1) \mathcal{B}$ for any $1 \leq a \leq \varkappa$ and that

$$
\sum_{a=1}^{\varkappa}\left(j_{1+a}-j_{a}\right)=N \text {. }
$$

There exists a $p$ with $1 \leq p \leq \varkappa$ such that

$$
j_{1+p}-j_{p}=\max _{1 \leq a \leq \varkappa}\left\{j_{1+a}-j_{a}\right\} .
$$

Let $J_{1}:=\left[j_{p}, j_{1+p}\right) \subset[0, N)$. Combining (4.28), (4.29), and the definition of $p$, we get for any $N \in \mathcal{F}$ that

$$
\begin{aligned}
\frac{\left|J_{1}\right|}{1+C_{1}} & =\frac{1}{1+C_{1}} \max _{1 \leq a \leq \varkappa}\left\{j_{1+a}-j_{a}\right\} \geq \frac{N}{\left(1+C_{1}\right) \varkappa} \\
& \geq \frac{N}{\left(1+C_{1}\right) \lambda(N)^{D-1}}
\end{aligned}
$$

and that

$$
\frac{\left|J_{1}\right|}{1+C_{1}}>N^{1-\mathcal{C}}
$$

by (4.6). Thus, we see for any sufficiently large $N \in \mathcal{F}$ that there exists a $\theta_{0}$ with

$$
\theta_{0} \in\left[\frac{\left|J_{1}\right|}{1+C_{1}}, \frac{C_{1}\left|J_{1}\right|}{1+C_{1}}\right) \cap \mathcal{B}
$$

by (4.5). Put

$$
M=M(N):=j_{p}+\theta_{0} \in\left[j_{p}+\frac{\left|J_{1}\right|}{1+C_{1}}, j_{p}+\frac{C_{1}\left|J_{1}\right|}{1+C_{1}}\right)
$$

and

$$
J_{2}:=\left[j_{p}, M\right) \subset J_{1} .
$$

Consequently, we get that $M \in D \mathcal{B}$ by $j_{p} \in(D-1) \mathcal{B}$ and $\theta_{0} \in \mathcal{B}$. 
Lemma 4.5. Assume that $N \in \mathcal{F}$ is sufficiently large. Then, for any $R \in J_{2}$, we have

$$
Y_{R}>0 \text {. }
$$

Proof. We verify Lemma 4.5 by induction on $R$. First we show that $Y_{M-1}>0$. Observe that

$$
\begin{aligned}
Y_{M-1}= & \sum_{\boldsymbol{k} \in \Gamma} B_{\boldsymbol{k}} \sum_{m=1}^{\infty} \beta^{-m} \rho(\boldsymbol{k} ; m+M-1) \\
= & \sum_{m=1}^{j_{1+p}-M} \beta^{-m} \sum_{\boldsymbol{k} \in \Gamma} B_{\boldsymbol{k}} \rho(\boldsymbol{k} ; m+M-1) \\
& +\sum_{\boldsymbol{k} \in \Gamma} B_{\boldsymbol{k}} \sum_{m=1+j_{1+p}-M}^{\infty} \beta^{-m} \rho(\boldsymbol{k} ; m+M-1) \\
= & : S_{1}+S_{2} .
\end{aligned}
$$

We give upper bounds for $\left|S_{2}\right|$. By the definition of $M$ and (4.31), we have

$$
j_{1+p}-M \geq j_{1+p}-j_{p}-\frac{C_{1}\left|J_{1}\right|}{1+C_{1}}=\frac{\left|J_{1}\right|}{1+C_{1}}>N^{1-\mathcal{C}}
$$

for any sufficiently large $N \in \mathcal{F}$. For simplicity, put, for a nonnegative integer $n$,

$$
\zeta_{n}:=\beta^{n^{1-2 c}} .
$$

Thus, using Lemma 4.1 and $M \leq N$, we get

$$
\begin{aligned}
\left|S_{2}\right| & \leq \sum_{\boldsymbol{k} \in \Gamma}\left|B_{\boldsymbol{k}}\right| \sum_{m=1+j_{1+p}-M}^{\infty} \beta^{-m} \rho(\boldsymbol{k} ; m+M-1) \\
& \ll \sum_{\boldsymbol{k} \in \Gamma}\left|B_{\boldsymbol{k}}\right| \sum_{m=1+j_{1+p}-M}^{\infty} \beta^{-m} \zeta_{m+M-1} \\
& \ll \sum_{m>N^{1-\mathcal{C}}} \beta^{-m} \zeta_{m+M-1} \leq \sum_{m>N^{1-\mathcal{C}}} \beta^{-m} \zeta_{m+N} .
\end{aligned}
$$

Since

$$
\lim _{m \rightarrow \infty}\left((m+1)^{1-2 \mathcal{C}}-m^{1-2 \mathcal{C}}\right)=0
$$

by $0<2 \mathcal{C}<1$ and the mean value theorem, we have

$$
\lim _{m \rightarrow \infty} \frac{\zeta_{m+1}}{\zeta_{m}}=1 .
$$


In particular, if $m$ is sufficiently large, then

$$
\frac{\zeta_{m+1}}{\zeta_{m}}<\frac{\beta+1}{2} \text {. }
$$

Hence,

$$
\begin{aligned}
\left|S_{2}\right| & \ll \beta^{-N^{1-\mathcal{C}}} \zeta_{2 N} \sum_{n=0}^{\infty} \beta^{-n}\left(\frac{\beta+1}{2}\right)^{n} \\
& \ll \beta^{-N^{1-2 C}}=o(1)
\end{aligned}
$$

as $N \in \mathcal{F}$ tends to infinity because we see

$$
-N^{1-\mathcal{C}}+(2 N)^{1-2 \mathcal{C}}<-N^{1-2 \mathcal{C}}
$$

for any sufficiently large $N \in \mathcal{F}$. In particular, if $N \in \mathcal{F}$ is sufficiently large, then

$$
\left|S_{2}\right|<\frac{1}{2} B_{\boldsymbol{g}} \beta^{-1}
$$

by $B_{\boldsymbol{g}}>0$.

On the other hand, we see for any $1 \leq m \leq j_{1+p}-M$ that $m+M-1 \notin(D-1) \mathcal{B}$ by $m+M-1 \in\left(j_{p}, j_{1+p}\right)$ and the definition of $j_{1}, \ldots, j_{1+\varkappa}$. Using Lemma 4.4 , we obtain

$$
\begin{aligned}
S_{1} & \geq \sum_{m=1}^{j_{1}+p-M} \beta^{-m}\left(B_{\boldsymbol{g}} \rho(\boldsymbol{g} ; m+M-1)-\sum_{\boldsymbol{k} \in \Gamma \backslash\{\boldsymbol{g}\}}\left|B_{\boldsymbol{k}}\right| \rho(\boldsymbol{k} ; m+M-1)\right) \\
& \geq \frac{1}{2} B_{\boldsymbol{g}} \sum_{m=1}^{j_{1+p}-M} \beta^{-m} \rho(\boldsymbol{g} ; m+M-1) \geq \frac{1}{2} B_{\boldsymbol{g}} \beta^{-1} \rho(\boldsymbol{g} ; M) \geq \frac{1}{2} B_{\boldsymbol{g}} \beta^{-1}
\end{aligned}
$$

by $M \in D \mathcal{B}$. Therefore, combining (4.32), (4.34), and (4.35), we deduce for any sufficiently large $N \in \mathcal{F}$ that

$$
Y_{M-1}>0
$$

Next we assume that $Y_{R}>0$ for certain $R \in\left(j_{p}, M\right)$. Then

$$
\begin{aligned}
Y_{R-1} & =\sum_{\boldsymbol{k} \in \Gamma} B_{\boldsymbol{k}} \sum_{m=1}^{\infty} \beta^{-m} \rho(\boldsymbol{k} ; m+R-1) \\
& =\frac{1}{\beta} \sum_{\boldsymbol{k} \in \Gamma} B_{\boldsymbol{k}} \rho(\boldsymbol{k} ; R)+\frac{1}{\beta} \sum_{\boldsymbol{k} \in \Gamma} B_{\boldsymbol{k}} \sum_{m=2}^{\infty} \beta^{-(m-1)} \rho(\boldsymbol{k} ; m-1+R) \\
& =: S_{1}^{\prime}+S_{2}^{\prime} .
\end{aligned}
$$


Lemma 4.4 implies that $S_{1}^{\prime} \geq 0$ because $R \notin(D-1) \mathcal{B}$ by $R \in\left(j_{p}, j_{1+p}\right)$. Moreover, it is easily seen that

$$
S_{2}^{\prime}=\frac{1}{\beta} \sum_{\boldsymbol{k} \in \Gamma} B_{\boldsymbol{k}} \sum_{m=1}^{\infty} \beta^{-m} \rho(\boldsymbol{k} ; m+R)=\frac{1}{\beta} Y_{R}>0 .
$$

Hence, (4.36) implies that $Y_{R-1}>0$. Therefore, we proved Lemma 4.5.

Applying (4.27), we get

$$
\operatorname{Card}\left(J_{2} \cap D \mathcal{B}\right) \leq \lambda(N)^{D} .
$$

Put

$$
\left(J_{2} \cap D \mathcal{B}\right) \cup\left\{j_{p}\right\}=:\left\{j_{p}=i_{1}<i_{2}<\ldots<i_{\omega}\right\},
$$

where

$$
\omega \leq 1+\lambda(N)^{D} \leq 2 \lambda(N)^{D}
$$

Let $i_{1+\omega}:=M$. Then there exists a $q$ with $1 \leq q \leq \omega$ such that

$$
i_{1+q}-i_{q}=\max _{1 \leq h \leq \omega}\left\{i_{1+h}-i_{h}\right\} \text {. }
$$

Put $I_{1}:=\left[i_{q}, i_{1+q}\right) \subset J_{2}$. For any $k$ with $1 \leq k \leq D$

$$
\left(i_{q}, i_{1+q}\right) \cap k \mathcal{B} \subset\left(i_{q}, i_{1+q}\right) \cap D \mathcal{B}=\varnothing
$$

by $(4.13)$.

We now estimate lower bounds for $\left|I_{1}\right|$. By the definition of $q$, we see

$$
\left|I_{1}\right|=\max _{1 \leq h \leq \omega}\left\{i_{1+h}-i_{h}\right\} \geq \frac{\left|J_{2}\right|}{\omega} .
$$

Combining (4.37), the definition of $M$, and (4.30), we obtain for any sufficiently large $N \in \mathcal{F}$ that

$$
\begin{aligned}
\left|I_{1}\right| & \geq \frac{1}{2 \lambda(N)^{D}} \cdot\left(M-j_{p}\right) \geq \frac{1}{2 \lambda(N)^{D}} \cdot \frac{\left|J_{1}\right|}{1+C_{1}} \\
& \geq \frac{N}{2\left(1+C_{1}\right) \lambda(N)^{2 D-1}}>2 N^{1-\mathcal{C}}
\end{aligned}
$$

by $(4.6)$.

For the proof of Theorem 3.2, it suffices to show that there exists a positive integer $R$ satisfying (4.12). Thus, we only have to show the following: 
Lemma 4.6. Assume that $N \in \mathcal{F}$ is sufficiently large. Then, for any $R$ with

$$
i_{q} \leq R<i_{q}+\frac{1}{2}\left|I_{1}\right|,
$$

we have

$$
0<Y_{R}<C_{5} \beta^{-R^{1-2 C}} .
$$

Proof. By Lemma 4.5 it suffices to show that $\left|Y_{R}\right|<C_{5} \beta^{-R^{1-2 C}}$ for any $R$ satisfying (4.40) under the assumption that $N$ is sufficiently large. In fact, if $R$ satisfies (4.40), then $R \in J_{2}$.

For an integer $R$ with (4.40), we estimate upper bounds for

$$
\left|Y_{R}\right|=\left|\sum_{\boldsymbol{k} \in \Gamma} B_{\boldsymbol{k}} \sum_{m=1}^{\infty} \rho^{-m} \rho(\boldsymbol{k} ; m+R)\right| .
$$

Let $\boldsymbol{k} \in \Gamma$. Take an integer $m$ with $1 \leq m<i_{1+q}-R$. Then we have $m+R \in\left(i_{q}, i_{1+q}\right)$. Thus, (4.38) implies that $\rho(\boldsymbol{k} ; m+R)=0$ for such an $m$. Using Lemma 4.1 and $R \leq N$, we get

$$
\begin{aligned}
\left|Y_{R}\right| & \leq \sum_{\boldsymbol{k} \in \Gamma}\left|B_{\boldsymbol{k}}\right| \sum_{m=i_{1+q}-R}^{\infty} \beta^{-m} \rho(\boldsymbol{k} ; m+R) \\
& \ll \sum_{\boldsymbol{k} \in \Gamma}\left|B_{\boldsymbol{k}}\right| \sum_{m=i_{1+q}-R}^{\infty} \beta^{-m} \zeta_{m+R} \ll \sum_{m=i_{1+q}-R}^{\infty} \beta^{-m} \zeta_{m+N} .
\end{aligned}
$$

We see by (4.40) and (4.39) that

$$
i_{1+q}-R>i_{1+q}-i_{q}-\frac{1}{2}\left|I_{1}\right|=\frac{1}{2}\left|I_{1}\right|>N^{1-\mathcal{C}}
$$

and that

$$
\left|Y_{R}\right| \ll \sum_{m>N^{1-C}} \beta^{-m} \zeta_{m+N}
$$

Consequently, using (4.33), we obtain

$$
\left|Y_{R}\right| \ll \beta^{-N^{1-\mathcal{C}}} \zeta_{2 N} \sum_{n=0}^{\infty} \beta^{-n}\left(\frac{\beta+1}{2}\right)^{n} \ll \beta^{-2 N^{1-2 \mathcal{C}}} .
$$

Namely, there exists a $C_{8}$ independent of $R$ and $N$ satisfying

$$
\left|Y_{R}\right| \leq C_{8} \beta^{-2 N^{1-2 C}} .
$$


In particular, if $N \in \mathcal{F}$ is sufficiently large, then

$$
\left|Y_{R}\right|<C_{5} \beta^{-N^{1-2 \mathcal{C}}} \leq C_{5} \beta^{-R^{1-2 \mathcal{C}}},
$$

which implies Lemma 4.6.

Acknowledgements. I am grateful to Prof. Shigeki Akiyama for fruitful comments. I appreciate the referee for the detailed reviews and the fruitful comments. This work was supported by JSPS KAKENHI Grant Number 15 K17505.

\section{References}

1. Adamczewski, B., Transcendance $\ll$ à la Liouville $\gg$ de certains nombres réels, $C$. $R$. Acad. Sci. Paris 338 (2004), 511-514.

2. Adamczewski, B. and Faverjon, C., Chiffres non nuls dans le développement en base entière des nombres algébriques irrationnels, C. R. Acad. Sci. Paris 350 (2012), 1-4.

3. Bailey, D. H., Borwein, J. M., Crandall, R. E. and Pomerance, C., On the binary expansions of algebraic numbers, J. Théor. Nombres Bordeaux 16 (2004), $487-518$.

4. Borel, É., Sur les chiffres décimaux de $\sqrt{2}$ et divers problèmes de probabilités en chaîne, C. R. Acad. Sci. Paris 230 (1950), 591-593.

5. Bugeaud, Y., Distribution Modulo One and Diophantine Approximation, Cambridge Tracts in Math. 193, Cambridge, 2012.

6. Cijsouw, P. L. and Tijdeman, R., On the transcendence of certain power series of algebraic numbers, Acta Arith. 23 (1973), 301-305.

7. Corvaja, P. and Zannier, U., Some new applications of the subspace theorem, Compos. Math. 131 (2002), 319-340.

8. Kaneko, H., Algebraic independence of real numbers with low density of nonzero digits, Acta Arith. 154 (2012), 325-351.

9. Kaneko, H., Arithmetical properties of real numbers related to beta-expansions, Preprint.

10. Kaneko, H., On the beta-expansions of 1 and algebraic numbers for a Salem number beta, Ergodic Theory Dynam. Systems 35 (2015), 1243-1262.

11. Kaneko, H., On the number of nonzero digits in the beta-expansions of algebraic numbers, Rend. Sem. Math. Univ. Padova 136 (2016), 205-223, doi:10.4171/ RSMUP/136-14.

12. Mahler, K., Arithmetische Eigenschaften der Lösungen einer Klasse von Funktionalgleichungen, Math. Ann. 101 (1929), 342-366.

13. NishiokA, K., Algebraic independence by Mahler's method and $S$-unit equations, Compos. Math. 92 (1994), 87-110.

14. NishiokA, K., Algebraic independence of Mahler functions and their values, Tohoku Math. J. 48 (1996), 51-70.

15. NishiokA, K., Conditions for algebraic independence of certain power series of algebraic numbers, Compos. Math. 62 (1987), 53-61. 
16. NishiokA, K., Mahler Functions and Transcendence, Lecture Notes in Math. 1631, Springer, 1996.

17. RÉNYI, A., Representations for real numbers and their ergodic properties, Acta Math. Acad. Sci. Hung. 8 (1957), 477-493.

18. Schmidt, W. M., Simultaneous approximation and algebraic independence of numbers, Bull. Amer. Math. Soc. 68 (1962), 475-478.

19. Shiokawa, I., Algebraic independence of certain gap series, Arch. Math. 38 (1982), $438-442$.

20. Tanaka, T., Algebraic independence of power series generated by linearly independent positive numbers, Results Math. 46 (2004), 367-380.

21. Tanaka, T., Algebraic independence of the values of power series generated by linear recurrences, Acta Arith. 74 (1996), 177-190.

Kaneko Hajime

Institute of Mathematics

University of Tsukuba

1-1-1 Tennodai

Tsukuba

Ibaraki, JP-305-8571

Japan

and

Center for Integrated Research in Funda-

mental Science and Engineering (CiRfSE)

University of Tsukuba

Tsukuba

Ibaraki, JP-305-8571

Japan

kanekoha@math.tsukuba.ac.jp

Received November 1, 2016

in revised form March 12, 2017 